\title{
Higgs production enhancement in P-P collisions using Monte Carlo techniques at $\sqrt{s}=13 \mathrm{TeV}$
}

\author{
M.H.M. Soleiman ${ }^{1, a}$, S.S. Abdel-Aziz ${ }^{1}$, and M.S.E. Sobhi ${ }^{1}$ \\ ${ }^{1}$ Cairo University, Faculty of Science, Physics Department, Giza, Egypt \\ 2 CTP, British University in Egypt, Egypt
}

\begin{abstract}
A precise estimation of the amount of enhancement in Higgs boson production through pp collisions at ultra-relativistic energies throughout promotion of the gluon distribution function inside the protons before the collision is presented here. The study is based mainly on the available Monte Carlo event generators (PYTHIA 8.2.9, SHERPA 2.1.0) running on PCs and CERNX-Machine, respectively, and using the extended invariant mass technique. Generated samples of 1000 events from PYTHIA 8.2.9 and SHERPA, 2.1.0 at $\sqrt{s}=13 \mathrm{TeV}$ are used in the investigation of the effect of replacing the parton distribution function $(P D F)$ on the Higgs production enhancement. The CTEQ66 and MSRTW2004nlo parton distribution functions are used alternatively on PYTHIA 8.2.9 and SHERPA 2.1.0 event generators in companion with the effects of allowing initial state and final state radiations (ISR and FSR) to obtain evidence on the enhancement of the SM-Higgs production depending on the field theoretical model of SM. It is found that, the replacement of PDFs will lead to a significant change in the SM-Higgs production, and the effect of allowing or denying any of ISR or FSR is sound for the two event generators but may be unrealistic in PHYTIA 8.2.9.
\end{abstract}

\section{Introduction}

In 1964, a boson field was theorized by Robert Brout, Franois Englert, Peter Higgs, Gerald Guralnik, C. R. Hagen, and Tom Kibble called Higgs boson $H^{0}$ to explain how the elementary particles in the $S M$ acquire their masses via the Higgs mechanism. In order to find a connection between the experiment and the theoretical models, a wave function had to be introduced to describe the hadrons in terms of its constituent partons using what is called the Factorization theory [1], which gives a prediction for the cross sections by factorizing out long-distance effects (which are not perturbatively calculable) from short-distance effects (which are perturbatively calculable) in a systematic fashion. The long distance effects are outlined into functions describing the distributions of partons in hadrons, where these functions are called the parton distribution functions $(P D F)$ [2]. It can be measured experimentally by finding common functions that fit all the data concerning many observations [3]. Many analysis approaches have been introduced to study these functions for data from lepton deep inelastic scattering reactions (either through electron scattering or neutrino scattering [4]) by suggesting suitable parametrizations of the structure functions, characterizing the structure of the target nucleon to leptonic probes [5]. Other models are introduced to describe the evolution of the parton density distributions with the square of the exchanged momentum $Q^{2}$, taking into account their physical dependence on

a e-mail: mhm.soleiman@gmail.com; mhmsoleiman@cu.edu.eg gluons, quarks, and antiquarks and deducing what is called the DGLAP equation [6].

On July 31, 2012, ATLAS [7] and CMS [8] experiments at the Large Hadron Collider (LHC) at CERN announced the discovery of the Higgs boson with a mass of $125.09 \pm 0.21 \mathrm{GeV}$, where the most significant channels for the detection are $H \rightarrow \gamma \gamma$ and $H \rightarrow Z Z \rightarrow$ $4 l$, since all final state particles can be measured very precisely to reconstruct the invariant mass, $m_{H}$, with good identification resolution. This discovery confirmed the validity of the standard model for describing elementary particles and their interactions. A clear signal for the Higgs mass in the diphoton channel for the entire data set collected by the $C M S$ experiment in $P P$ collisions during 2011 and 2012 LHC running periods, gives the invariant mass of $124.7 \pm 0.34 \mathrm{GeV}$ with the significance of $5.7 \sigma$, where $g g H$ process is expected using next-toleading order ( $N L O)$ matrix elements [9].

The parton distribution functions multiply the partonparton scattering cross section with the summation over all parton families to formulate the total scattering cross section of the hadron-hadron interactions. The parton structure of hadrons in QCD, drives a rapid increase in the proton-proton scattering cross section at high energies [10]. The possibility of enhancement of heavy boson production through hadron-hadron collision has been studied theoretically for $W^{ \pm}, Z$ bosons at the $L H C$ [11]. The study assumed that the standard model parameters are fixed at standard values and the PDFs of MRST99 and CTEQ5 started at scale of $Q^{2}=1 \mathrm{GeV}^{2}$. The nonnegligible variations in the production cross section have 
been predicted. The initial state radiation (ISR) and final state radiation $(F S R)$ must be taken into consideration in constructing the Higgs invariant mass. The study of the effects of $I S R / F S R$ on the reconstruction of heavy lepton (top quark) masses are studied in [12] using PYTHIA 8 and POWHEG 1.0 Monte Carlo event generators. ISR and FSR contribute to the systematic uncertainty of the estimation of the number of Higgs events in association with $W^{ \pm}, Z$ bosons by about $5.2 \%$ in the search for Higgs boson production [13]. ISR/FSR modeling is vital in the reconstruction of Jets and photons at $C M S$. While the inclusive analysis is useful for constraining PDFs, the more exclusive channel could probe details of ISR and FSR modeling [14]. Search for the Higgs boson in all the hadronic final state using the full $C D F$ data set taking into account the effects of uncertainties from ISR and FSR is done in Ref. $[15,16]$.

In this work, we study the Higgs production in the gluon channel $g g \rightarrow H^{0}$ through the diphoton decay $\left(H^{0} \rightarrow \gamma \gamma\right)$ using different parton distribution functions $(P D F s)$ available already in Monte Carlo event generators (PYTHIA 8.2.9 and SHERPA 2.1.0). The first PDF is named CTEQ66 and expresses two $p p$ collisions in excited proton states and the second is identified as MST RW2004nlo and describes pp collision in normal proton states.

\subsection{The discovery channel $H^{0} \rightarrow \gamma \gamma$}

The decay mode $H^{0} \rightarrow \gamma \gamma$ is very relevant to experimental searches for the Higgs boson. Although having a small branching ratio (about $0.23 \%$ ) [17], it has a clean signature and lower background than the other decay modes. The energy and momentum of the photons can be measured very precisely and an accurate reconstruction of the invariant mass of the decayed particle could be obtained by a simple calculation algorithm.

In the $H^{0} \rightarrow \gamma \gamma$ channel a search is performed for a narrow peak over a smoothly falling background in the invariant mass distribution of two high $p_{T}$ photons. The decay mode can be well identified experimentally but the signal rate is small compared to the backgrounds both coming from two prompt photons (irreducible background) and from those in which one or more of the photons are due to decay products or misidentified particles in jets (reducible background). In order to optimize search sensitivity and separate the various Higgs production modes, ATLAS and CMS experiments split events into several mutually exclusive categories. Diphoton events containing a high $p_{T}$ muon, electron, dijets, or missing energy consistent with the decay of a $W$ or $Z$ boson are tagged in the vector boson to Higgs $(V H)$ production category, and those containing energetic dijets with a large mass and pseudorapidity difference are assigned to the vector boson fusion $(V B F)$ production category and the remaining events (99\% of the total) are considered in the gluon fusion production $(g F)$ category. While the $V H$ category is relatively pure, the $V B F$ category has significant contamination from the gluon fusion process. ATLAS uses the diphoton transverse momentum orthogonal to the diphoton thrust axis in the transverse plane [17].
Table 1. The $x$ and $Q^{2}$ ranges of the grids for two $P D F s$ and the order of the running of $\alpha_{s}$ at the value of $M_{Z}$.

\begin{tabular}{|l|c|c|c|c|}
\hline$P D F$ & $x$ range & $Q^{2}$ range $\left(\mathrm{GeV}^{2}\right)$ & $\alpha_{s}$ & $\alpha_{s}\left(M_{Z}\right)$ \\
\hline$M S T W$ & $10^{-6}-1$ & $1.00-10^{9}$ & $N L O$ & 0.12018 \\
CTEQ66 & $10^{-8}-1$ & $1.69-10^{10}$ & $N L O$ & 0.1180 \\
\hline
\end{tabular}

\subsection{PDFs available in monte-carlo event simulations}

Most of the MonteCarlo generators offers several PDFs with different orders; leading order $(L O)$, next-to-leading order $(N L O)$ and some next-to-next leading order $(N N L O)$, where the higher orders PDFs give more precise results of the cross sections as the partons inside the proton are allowed to have a total momentum larger than the momentum of the proton [18]. The effect of some of the next-to-leading-order corrections permits a large value of the gluon distribution function at small scale parameter $x$ without compromising the quark distributions at large $x$. Two separate groups obtained full sets of parton distributions by fitting the structure function with data. The $M C$-adapted $P D F$ s released so far from the $M R S T$ group with $L O$ and $N L O$ corrections [18], and from the $C T E Q$ group with $M C 1, M C 2$ and $M C S$ [19], where the most basic features of each $P D F$ are listed in Table 1.

- MST R - PDFs: These PDFs have been introduced by the $M S T W$ group. Many releases are available based on different fits from several experiments. The group produces $P D F s$ at $L O, N L O$ and NNLO.

- $C T E Q-\boldsymbol{P D F}$ : These PDFs have been introduced by the $C T E Q$ group, adopting an approach, which is very similar to that of $M S T W$. The groups fit the majority of the available data, the recent significant update in widest use is CTEQ66 [20]. This is slightly older than the MSTW2008 sets and is based on Tevatron data. They are available at NLO .

\subsection{Diphoton reconstruction in MONTE CARLO analysis}

In our analysis, we introduce the algorithms followed to reconstruct the Higgs invariant mass from photon candidates using two different Monte Carlo generators: PYTHIA 8.2.9 [21] and SHERPA 2.1.0 Monte Carlo generator [22] at $\sqrt{s}=13 \mathrm{TeV}$. The algorithms do not make any hypothesis as to whether the photons originating from the Higgs particle (i.e Assuming $m_{H}=125 \mathrm{GeV}$ according to the latest updates from the $L H C$ ) or not, and without any selection criteria. The reconstruction process in any event is based on the collection of all possible photon candidates available in that event without any restrictions on the original parent particle.

The parton distribution functions $(P D F s)$ available in both PYTHIA 8.2.9 and SHERPA 2.1.0 Monte Carlo generators are based on the calculations and fits done by the $M S T W$ group and the $C T E Q$ group. Our selections are MRSTW2004nlo and CTEQ66. These PDFs are already available officially through the programmatic library $L H A P D F[23]$.

As shown in Fig. 1 the diphoton decay mode is mediated by loop diagrams containing charged particles. The topquark loop and the $W^{ \pm}$boson loop diagrams dominate the decay amplitude, though they contribute with 


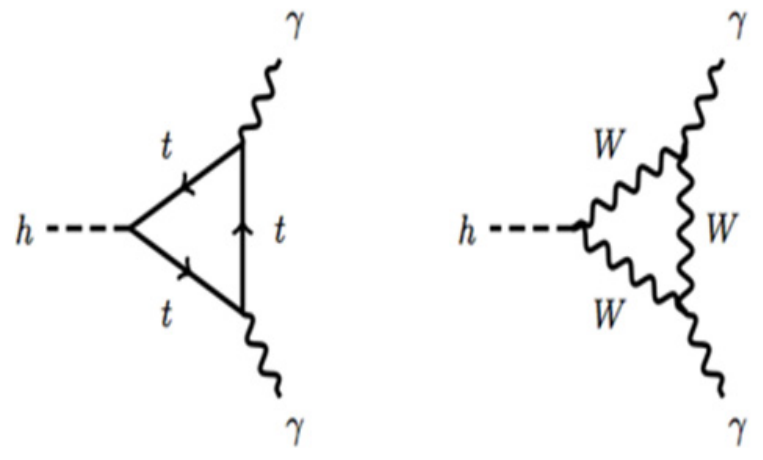

Figure 1. The Feynman diagram for Higgs decays into diphoton: (Left) topquark loop (Right) $W^{ \pm}$boson loop.

opposite signs. In PYTHIA 8, the multiple interaction (MI) process $f \vec{f} \rightarrow W^{ \pm} W^{\mp}$ (mainly $f=\tau, b, t, c$ ) have been chosen to be the interference process. This process goes through weak double Boson exchange.

In SHERPA 2.1.0, the shown process for Higgs production is the inclusive gluon fusion. The inclusive process is calculated at next-to-leading order (NLO) accuracy, including all interference effects between Higgs-boson production and the $S M$ channel $g g \rightarrow \gamma \gamma$ contributions with all partonic processes are allowed. The corresponding matrix elements are taken from [24] and [25].

\subsection{Effect of initial state radiations and final state radiations}

The parton showers in Monte Carlo generators are subdivided into two types, initial-state radiation (ISR) and final-state radiation (FSR). Initial-state radiation (ISR) arises because the incoming charged particles can radiate before entering the hard scattering process. A constituent parton from each of the incoming hadrons starts at high energy with low virtuality and evolves to higher spacelike virtuality by emitting partons and losing energy. The showering of these partons terminates when they collide to initiate the hard subprocess, and that is why $I S R$ is considered to be complicated. It is ordered in increasing virtuality but since the simulation starts with the hard interaction, a corresponding backward-evolutions algorithm was formulated by Schumann and Krauss [26] and adopted in all shower models. The key point in backward evolution is that the evolution probability depends on the amount of partons that could have given rise to the one being evolved. As the collision energy increases, partons at smaller scale parameter $x$ become involved and more particles can be produced in the ISR (see Fig. 2).

The Final-state radiation $(F S R)$ starts after the hard collision as it takes the products of the collision and describes how they branch into more particles. After the FSR the hadronization starts, and the partons produced in the previous stages split up and combine into colorless hadrons than can survive and possibly reach a detector. As shown in Fig. 2, the ISR are competing processes and can modify the total energy by further ISR branching, while $F S R$ redistributes the energy among the outgoing partons. That is why $I S R$ can lead to changes in the incoming $P D F s$. In the case of FSR, a color dipole can stretch from a

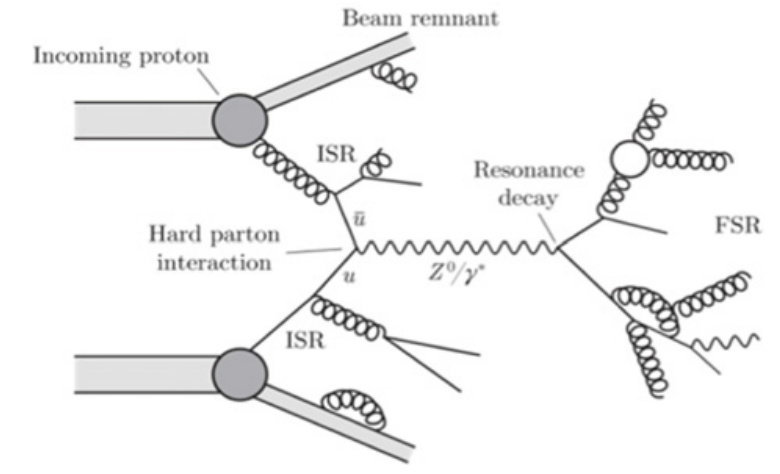

Figure 2. The Feynman diagram for Higgs decays into diphoton: (Left) topquark loop (Right) $W^{ \pm}$boson loop.

radiating parton to a beam remnant, leading to momentum shuffling between the beam and the parton. ISR can result in large $x$ values taken from the beams, and leading to flavor changes in the PDFs.

\section{Event generation and analysis}

The aim of the study is to prove the effect of $P D F$ on the Higgs production in $p p$ collision at $\sqrt{s}=13 \mathrm{TeV}$. The invariant mass plot for the Higgs decay into diphoton has been constructed using two different Monte Carlo event generators PYTHIA 8.2.9 and SHERPA 2.1.0. The diphoton invariant mass distribution is performed over the range $0<m_{\gamma \gamma}<300 \mathrm{GeV}$. Binned fits are used for the speed of computation, and the bin size chosen is $1200 \mathrm{MeV}$, which is sufficiently small compared to the mass resolution, so that no information is lost. The description of the expected peak of Higgs invariant mass $m_{\gamma \gamma}$ is executed for the invariant mass region $100 \leq$ $m_{\gamma \gamma} \leq 150 \mathrm{GeV}$. The suitable fitting functions describing the Higgs peak, the background, and the total invariant mass distribution are given as follows:

$$
f_{1}(x ; p[1], p[2], p[3])=(p[1]+p[2] x) e^{-p[3] x}
$$

where $f_{1}(x ; p[1], p[2], p[3])$ is exponential times polynomial function.

$$
\begin{aligned}
f_{2}(x ; p[4], p[5], p[6]) & =p[4] e^{-e^{-z}-z+1} ; \\
z & =(x-p[5]) / p[6]
\end{aligned}
$$

where $f_{2}(x ; p[4], p[5], p[6])$ is non symmetric extreme peak function.

$$
\begin{aligned}
f_{\text {total }}(x ; p[1] \ldots p[6])= & f_{1}(x ; p[1], p[2], p[3]) \\
& +f_{2}(x ; p[4], p[5], p[6])
\end{aligned}
$$

where $f_{\text {total }}(x ; p[1], \ldots, p[6])$ is the total fitting function.

These fitting functions are found to be the best functions describing the background and the Higgs peak in the invariant mass plot inside the $m_{\gamma \gamma}$ region from 100 to $150 \mathrm{GeV}$.

The invariant mass extended technique is:

1. Obtain the invariant mass plot of $m_{\gamma \gamma}$ for a sample of (say 50 events) of PYTHIA 8.2.9 and SHERPA 2.1.0 events and for two predetermined PDFs.

2. Fit the invariant mass spectrum in the region $100 \leq$ $m_{\gamma \gamma} \leq 150 \mathrm{GeV}$ with the total fitting function $f_{\text {total }}$ to obtain the six fitting parameters. 


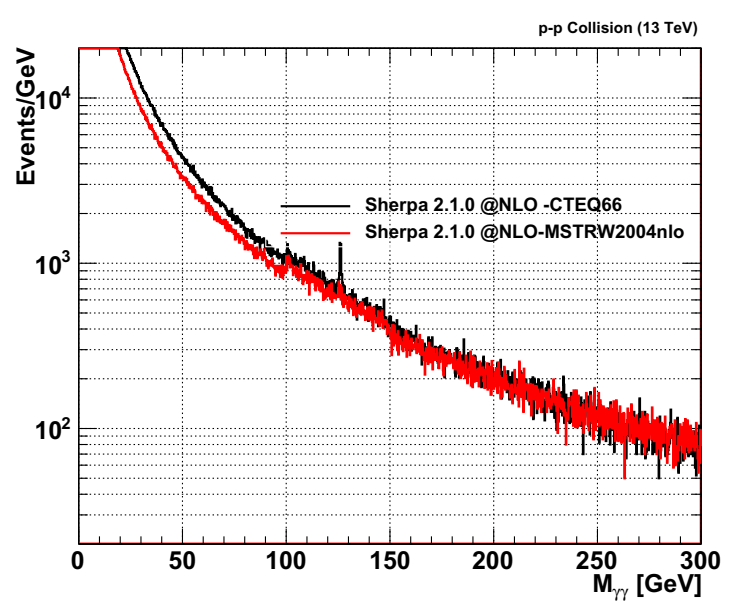

Figure 3. The diphoton invariant mass plot using SHERPA 2.0.1 for two PDFs (CTEQ66 and M RST W2004nlo).

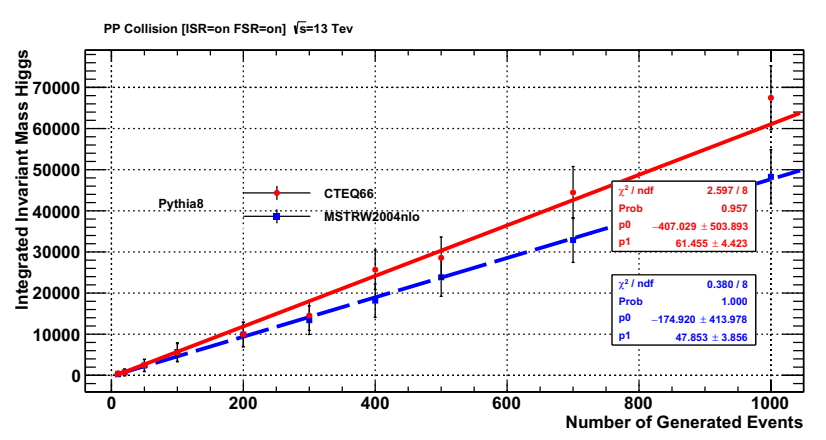

Figure 4. The number of generated events vs. the integrated invariant mass using PYTHIA 8.2.9. The error bars are scaled by factor 30 .

3. Subtract the integration of the exponential times polynomial fitting function from the invariant mass spectrum in the region $100 \leq m_{\gamma \gamma} \leq 150 \mathrm{GeV}$ to obtain a quantity proportional to the generated number of Higgs.

4. Obtain a plot between the integrated number of generated Higgs bosons and the number of generated events.

5. Determine the slope of the line fitting the points on the plot from the previous step.

The slopes of the fitting lines in the obtained graphs are sensitive to the replacement of PDFs. We take the error bars to be calculated by the square root of the integrated number of Higgs bosons from the invariant mass plot using the above technique. The calculation, fittings, and plots are produced on ROOT 5.34 environment.

\section{Results and discussion}

The diphoton invariant mass distributions are performed over the range $0<m_{\gamma \gamma}<300 \mathrm{GeV}$. Binned fits are used for speed of computation, and the bin size chosen to be $1200 \mathrm{MeV}$, is sufficiently small compared to the mass resolution that no information is lost (see Fig. 3).

Table 2, shows the fit parameters of this combined functions for 1000 events generated by SHERPA 2.1.0.

The invariant mass spectrum shown in Fig. 3, has been produced with different number of events (i.e. 1000, 700, 600, 50, 30, 30, 10), with the two different PDFs. The

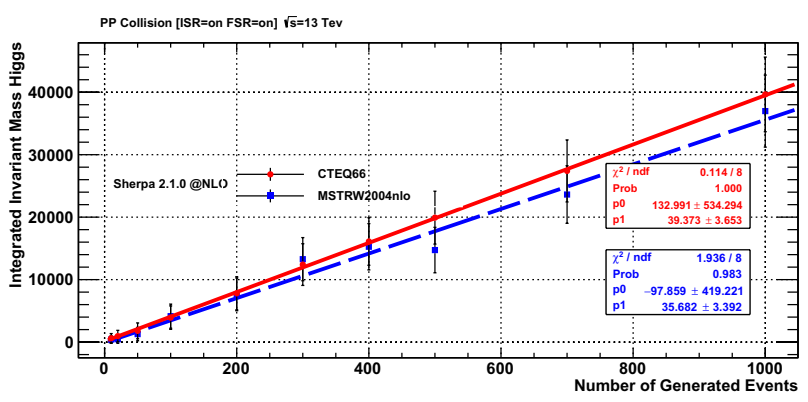

Figure 5. The number of generated events vs. the integrated invariant mass using SHERPA 2.1.0. The error bars are scaled by factor 30 .

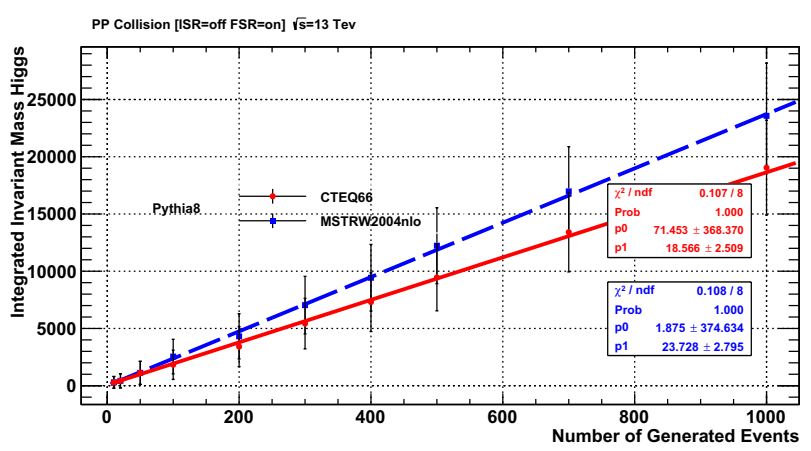

Figure 6. The number of generated events vs. the integrated invariant mass using PYTHIA 8.2.9 with ISR off. The error bars are scaled by factor 30 .

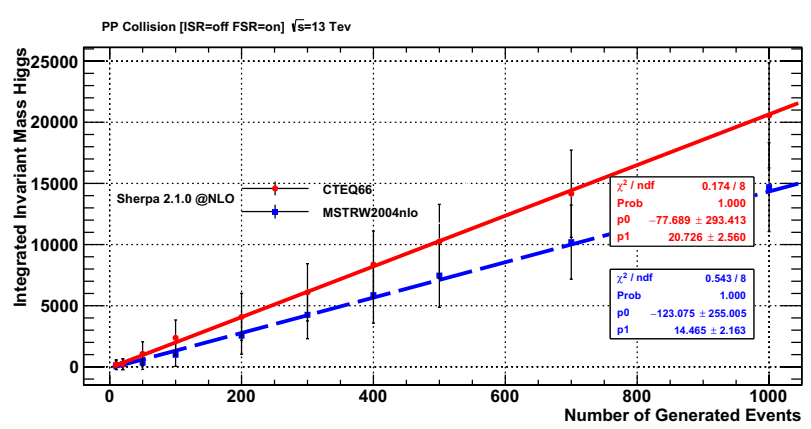

Figure 7. The number of generated events vs. the integrated invariant mass using SHERPA 2.1.0 with ISR off. The error bars are scaled by factor 30 .

integration under the peak signal around $126 \mathrm{GeV}$ has been estimated. Figures 4 and 5 show the relation between the number of generated events vs. the integrated invariant mass for PYTHIA 8.2.9 and SHERPA 2.1.0 respectively.

The two plots show a linearity between the number of generated events and the number of Higgs events calculated by the integration under the peak signal, where the slope of each straight line shows an improvement in case of CTEQ66 than MSTRW2004nlo. The values for these straight line parameters are shown in Table 3, where $P[0]$ is the y-intercept while $p[1]$ is the slope of each line.

Due to the color-charged partonic initial states, every hard process at hadron colliders is accompanied by initial- and subsequent final-state radiation [8]. The results presented are obtained by keeping the initial state radiation off (see Figs. 6 and 7) and the final state radiation off (see Figs. 8 and 9). 
Table 2. The fit parameter for the diphoton invariant mass plot using SHERPA 2.1.0.

\begin{tabular}{|c|c|c|c|c|c|}
\hline$P[1]$ & $P[2]$ & $P[3]$ & $P[4]$ & $P[5]$ & $P[6]$ \\
\hline 60 & 126 & $4.2 E-02$ & $1.59993 E+03$ & $-3.2952 E-03$ & $1.42408 E-02$ \\
\hline
\end{tabular}

Table 3. The fit parameter for the integrated invariant mass for different number of events in the case of ISR on and FSR on.

\begin{tabular}{|l|c|c|c|c|}
\hline PDF & Monte Carlo generator & $p[0]$ & $p[1]$ & $\chi^{2} / n . d . f$ \\
\hline CTEQ66 & PYTHIA 8.2.9 & $-407.029 \pm 503.893$ & $61.455 \pm 4.423$ & $2.597 / 8$ \\
MST RW2004nlo & PYTHIA 8.2.9 & $-174.920 \pm 413.978$ & $47.853 \pm 3.856$ & $0.380 / 8$ \\
CTEQ66 & SHERPA 2.1.0 & $132.991 \pm 534.294$ & $39.373 \pm 3.653$ & $0.114 / 8$ \\
MST RW2004nlo & SHERPA 2.1.0 & $-97.859 \pm 419.221$ & $35.682 \pm 3.392$ & $1.936 / 8$ \\
\hline
\end{tabular}

Table 4. The fit parameter for the integrated invariant mass for different number of events in the case of ISR off and FSR on.

\begin{tabular}{|l|c|c|c|c|}
\hline PDF & Monte Carlo generator & $p[0]$ & $p[1]$ & $\chi^{2} / n . d . f$ \\
\hline CTEQ66 & PYTHIA 8.2.9 & $71.453 \pm 368.370$ & $18.566 \pm 2.509$ & $0.107 / 8$ \\
MST $R$ W2004nlo & PYTHIA 8.2.9 & $1.875 \pm 374.634$ & $23.728 \pm 2.795$ & $0.108 / 8$ \\
CTEQ66 & SHERPA 2.1.0 & $-77.689 \pm 293.413$ & $20.726 \pm 2.560$ & $0.174 / 8$ \\
MST $R$ W2004nlo & SHERPA 2.1.0 & $-123.075 \pm 255.005$ & $14.465 \pm 2.163$ & $0.543 / 8$ \\
\hline
\end{tabular}

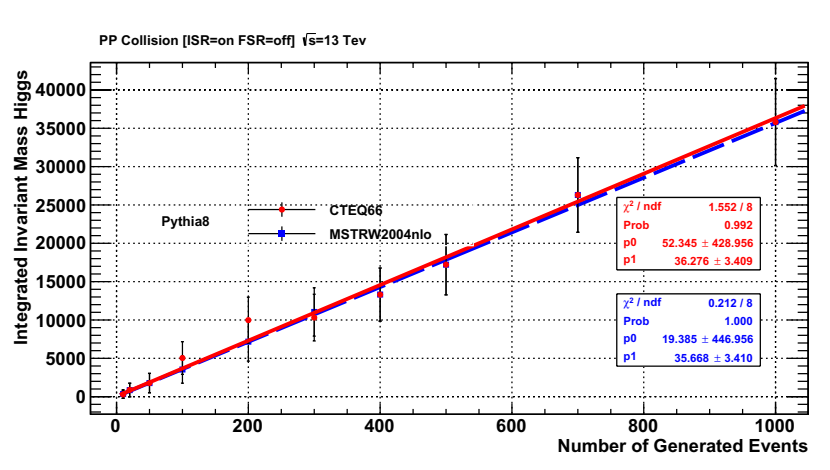

Figure 8. The number of generated events vs. the integrated invariant mass using PYTHIA 8.2.9 with FSR off. The error bars are scaled by factor 30 .

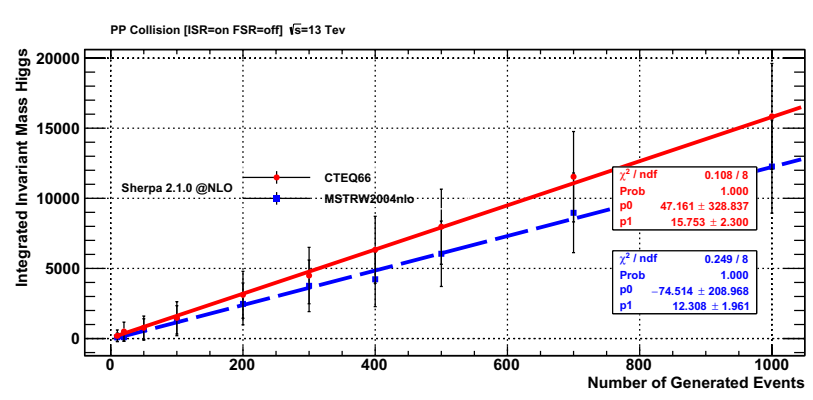

Figure 9. The number of generated events vs. the integrated invariant mass using SHERPA 2.1.0 with FSR off. The error bars are scaled by factor 30 .

As shown in Figs. 6 and 7, the predictions from SHERPA 2.1.0 do not match with the predictions given by PYTHIA 8.2.9 (See Table 4). The results obtained from PYTHIA 8.2.9 show an inversion in the Higgs integrated production, where CTEQ66 is expected to be higher than MSTRW2004nlo. The inversion appears in Fig. 6 represents that, PYTHIA 8.2.9 modifies the PDFs before starting the hard process.

Also, in Figs. 8 and 9, the predictions from SHERPA 2.1.0 do not match with the predictions given by PYTHIA 8.2.9 (See Table 5) when the final state radiation is off. The two graphs are consistent in the ordering of the

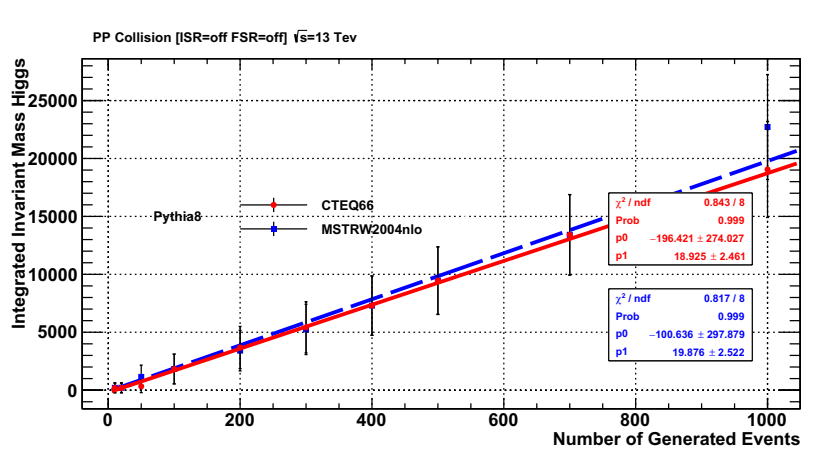

Figure 10. The number of generated events vs. the integrated invariant mass using PYTHIA 8.2.9 with ISR and FSR are off. The error bars are scaled by factor 30 .

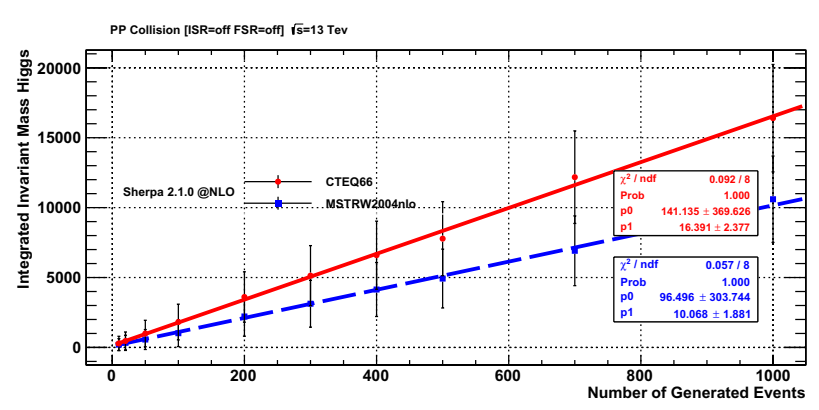

Figure 11. The number of generated events vs. the integrated invariant mass using SHERPA 2.1.0 with ISR and FSR are off. The error bars are scaled by factor 30 .

behaviors for the selected PDFs, but FSR is expected to be of minor effect on the Higgs production.

Table 5 gives the predictions from SHERPA 2.1.0 and PYTHIA 8.2.9 where FSR is off. In PYTHIA 8.2.9, CTEQ66 and MSTRW2004nlo give approximately the same results, while in SHERPA an enhancement can be observed with CTEQ66.

The ideal case study is where both $I S R$ and FSR are off. This case expresses the Higgs production enhancement including only the effects of replacing PDFs. Figures 10 and 11 show the results of the extended invariant mass technique obtained for PYTHIA 8.2.9 and SHERPA 2.1.0 where ISR and FSR are off. Table 6 gives the obtained 
Table 5. The fit parameter for the integrated invariant mass for different number of events in the case of ISR on and FSR off.

\begin{tabular}{|l|c|c|c|c|}
\hline PDF & Monte Carlo generator & $p[0]$ & $p[1]$ & $\chi^{2} / n . d . f$ \\
\hline CTEQ66 & PYTHIA 8.2.9 & $752.345 \pm 428.956$ & $36.276 \pm 3.409$ & $1.552 / 8$ \\
MST RW2004nlo & PYTHIA 8.2.9 & $19.385 \pm 446.956$ & $35.668 \pm 3.410$ & $0.212 / 8$ \\
CTEQ66 & SHERPA 2.1.0 & $47.161 \pm 328.837$ & $15.753 \pm 2.300$ & $0.108 / 8$ \\
MST RW2004nlo & SHERPA 2.1.0 & $-74.514 \pm 208.968$ & $12.308 \pm 1.961$ & $0.249 / 8$ \\
\hline
\end{tabular}

Table 6. The fit parameter for the integrated invariant mass for different number of events in the case of ISR off and FSR off.

\begin{tabular}{|l|c|c|c|c|}
\hline PDF & Monte Carlo generator & $p[0]$ & $p[1]$ & $\chi^{2} / n . d . f$ \\
\hline CTEQ66 & PYTHIA 8.2.9 & $-196.421 \pm 274.027$ & $18.925 \pm 2.461$ & $0.843 / 8$ \\
MST RW2004nlo & PYTHIA 8.2.9 & $-100.636 \pm 297.879$ & $19.876 \pm 2.522$ & $0.817 / 8$ \\
CTEQ66 & SHERPA 2.1.0 & $141.135 \pm 369.626$ & $16.391 \pm 2.377$ & $0.092 / 8$ \\
MST $R$ W2004nlo & SHERPA 2.1.0 & $96.496 \pm 303.744$ & $10.068 \pm 1.881$ & $0.057 / 8$ \\
\hline
\end{tabular}

fit parameters for this case study. In this last case, the inversion in the behavior is observed for the events generated by PYTHIA 8.2.9. The resolution between the two PDFs plots for PYTHIA 8.2.9 is very low in general, while it is clear in SHERPA 2.1.0.

\section{Conclusions}

In the present work we study the possibility of preevolution of parton distribution function with $Q^{2}$ to affect the production of Higgs bosons in the frame of $S M$. The sensitive method of integrated number of Higgs invariant mass is used to study the effect of replacing PDFs (CTEQ66 for enhanced gluon state and MSTRW2004nlo for ground state) in each of PYTHIA 8.2.9 and SHERPA 2.1.0 event generators.

The conclusions are given in the following:

1. The observation of the enhancement in the Higgs production is possible in the generated events from PYTHIA 8.2.9 and SHERPA 2.1.0. In the events where ISR and FSR are allowed, the replacement of PDFs shows significant differences in the Higgs production (The samples used in this study contain 1000 events for each event generator and for each PDFs).

2. The effect of replacing PDFs in PYTHIA 8.2.9 is more sound than in SHERPA 2.1.0 by a factor of three.

3. The expected behavior of the integrated number of Higgs invariant mass is accepted for all cases of $I S R$ and FSR in SHERPA 2.1.0 while in PYTHIA 8.2.9, the absence of ISR leads to an inversion of the behavior. This shows that, PYTHIA 8.2.9 modifies the gluon distributions before executing the hard process.

4. In the case of removing the effect of ISR and FSR, there is an inversion in the behavior for the Higgs production of the two PDFs in PYTHIA 8.2.9 and a suppression in the Higgs production for both PYTHIA 8.2.9 and SHERPA 2.1.0 event generators with good resolution in SHERPA 2.1.0.

Thanks are due to Cairo University, the research committee for partial funding the symposium attendance and encouraging the publishing of this work.

\section{References}

[1] D. E. Soper, J. C. Collins, and G. Sterman, Adv. Ser. Direct. High Energy Phys. 5, 91 (1989)

[2] D. A. Kosower, W. T. Giele, S. A. Keller, arXiv: hep-ph/0104052v1, 23 (2011)

[3] M. Dittmar, et al., arXiv:hep-ph/0511119, 160 (2005)

[4] Rein Dieter, et al., Annals Phys. 133, 106 (1981)

[5] Ta. Pie Cheng., Gauge Theory of Elementary Particle Physics, (Oxford University Press, ISBN 0-19851961-3, 1983)

[6] P. Kooijman, and J. Engelen, Progress in Particle and Nuclear Physics 41, 1-47 (1998)

[7] ATLAS Collaboration (G. Aad, et al.), Phys. Rev. Lett. 716, 1-29 (2012)

[8] CMS Collaboration (Chatrchyan, Serguei, et al.), Phys. Lett. B 716, 30-61 (2012), arXiv:[hepex]1207.7235, CMS-HIG-12-028, CERN-PH-EP2012-220

[9] CMS Collaboration (Khachatryan, Vardan, et al.) Eur. Phys. J. C 74(10), 3076 (2014), arXiv: [hep-ex] 1407.0558, CMS-HIG-13-001, CERNPH-EP-2014-117

[10] Loyal Durand, and Pi Hong, Phys. Rev. Lett. 58, 303 (1987)

[11] Martin, D. Alan, et al., Eur. Phys. J. C 14, 133-145 (2000), hep-ph/9907231, DTP-99-64, OUTP-99-31-P, RAL-TR-1999-047

[12] Valentina Tudorache, Mihai Cuciuc, Romanian Reports in Physics 64(4), 945-956 (2012)

[13] CDF Collaboration, CDF/ANAL/EXOTIC/PUBLIC, 8957 (2007)

[14] M. Voutilainen (for CMS Collaboration), Presented at EDS Blois Conference, Proceedings, 14th Workshop on Elastic and Diffractive Scattering (EDS Blois Workshop) on Frontiers of QCD (December 15-21, 2011)

[15] CDF Collaboration, Phys. lett. B 717, 1973 (2012)

[16] CDF Collaboration, Springer-Verlagh, JHEP 02, 004 (2013), ArXiv : 1208.6445

[17] LHC Higgs Cross Section Working Group (S. Heinemeyer), Handbook of LHC Higgs Cross Sections: 3. Higgs properties: Report of the LHC Higgs Cross Section Working Group, CERN, (2013)

[18] A. Sherstnev, and R. S. Thorne, arXiv:[hep-ph]0807.2132v1, (2008)

[19] H. L. Lai, J. Huston, et al., JHEP 04, 089 (2007) 
[20] Tomas Kasemets, Inclusion of parton distribution functions in PYTHIA 8, Master's thesis, Department of Astronomy and Theoretical Physics, Lund University (February 15, 2010)

[21] S. Mrenna, T. Sjöstrand, and P. Skands, Comput. Phys. Commun. 178, 852-867 (2008), arXiv : [hepph] 0710.3820, CERN-LCGAPP-2007-04, LU-TP07-28, FERMILAB-PUB-07-512-CD-T

[22] T. Gleisberg, et al., JHEP 0902, 07 (2009), arXiv:[hep-ph] 0811.4622, FERMILAB-PUB-08477-T, SLAC-PUB-13420, ZU-TH-17-08, DCPT-08-138, IPPP-08-69, EDINBURGH-200830, MCNET-08-14
[23] Andy Buckley, (LHAPDF collaboration), Eur. Phys. J. C75, 132 (2015), arXiv:1412.7420 [hep-ph] GLAS-PPE-2014-05, MCNET-14-29, IPPP-14-111, DCPT-14-222

[24] L. J. Dixon and Y. Li, Phys. Rev. Lett 111, 111802 (2013), arXiv: [hep-ph] 1305. 3854

[25] T. Sjöstrand, Phys. Lett. B157, 321-325 (1985), FERMILAB-PUB-85-023-T, Torbjörn Sjöstrand, et al.,Comput. Phys. Commun. 135, 238-259 (2001), arXiv: [hep-ph] 0010017, LU-TP-00-30

[26] S. Schumann, and F. Krauss, JHEP 0803, 038 (2008), arXiv:[hep-ph] 0709.1027 